\title{
Application of pervaporation and osmotic membrane distillation to the re- generation of spent solutions from the osmotic food dehydration
}

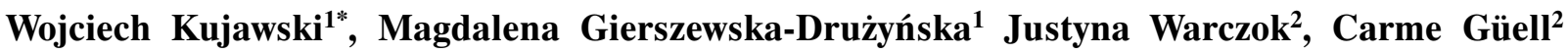 \\ ${ }^{1}$ Nicolaus Copernicus University, Faculty of Chemistry, ul. Gagarina 7, 87-100 Toruń, Poland \\ ${ }^{2}$ Rovira i Virgili University, Department of Chemical Engineering, Avda. Paisos Catalans 26, 43007 Tarragona, Spain \\ *Corresponding author: e-mail: kujawski@chem.umk.pl
}

\begin{abstract}
Results of pervaporation (PV) of sucrose and calcium chloride spent solutions were presented. Additionally, osmotic membrane distillation (OMD) of sucrose solutions was investigated. It was found that the regeneration of spent sucrose solution for the reuse is possible by using PV or OMD processes. However, OMD process produces another spent stream i.e. $\mathrm{CaCl}_{2}$. Pervaporation membranes showed fluxes in the range of $0.5-0.9 \mathrm{~kg} \mathrm{~m}^{-2} \mathrm{~h}^{-1}$ in contact with $40^{\circ}$ Brix sucrose solution, whereas OMD water permeate fluxes were in the range of $4-5 \mathrm{~kg} \mathrm{~m}^{-2} \mathrm{~h}^{-1}$ for the same feed concentration.

Two different hybrid processes were suggested: i) pretreatment followed by OMD reconcentration of spent sucrose solution and independently PV for $\mathrm{CaCl}_{2}$ regeneration; ii) membrane pretreatment (MP) followed by $\mathrm{PV}$ of sucrose solution. Based on the experimental results, the membrane areas for both systems were calculated and compared. MP-PV system seems to be a better solution for the spent mixtures management.
\end{abstract}

Keywords: food processing, spent solution management, pervaporation, osmotic membrane distillation.

\section{INTRODUCTION}

For the last few decades one of the most commonly used method of preservation and storage of fruit and vegetables was thermal drying. Nowadays is less applied, due to its adverse effect on the final taste, flavor and texture of the obtained product ${ }^{\mathbf{1}, 2}$.

This adverse influence is not observed in case of osmotic dehydration (OD), a well established method of food conservation used on a large scale in the food industry $^{3-5}$. OD process is based on the product immersion in a hypertonic solution of electrolyte or non-electrolyte ${ }^{6,7}$. The composition of a plant material is modified through a partial water removal and an impregnation by solutes (osmotic agent), without affecting its structural integrity (Fig. 1). The efficiency of the process and the quality of the final product are mainly affected by the osmotic agent properties. For that reason, the osmotic agent should exhibit several characteristic features. It must be nontoxic, showing also neutral influence on the taste and flavor of the final product ${ }^{8}$. Concentrated sucrose solutions, sodium and calcium chloride solutions, as well as binary or ternary solutions (e.g. sodium chloride with sucrose or/and glucose) are among commonly used osmotic agents (OA). Sorbitol, fructose and glucose solutions, starch syrup or hydrolyzed starch syrup are also used $^{6-8}$.

One of the determining factors of OD process application is necessity of the adequate amount of the hypertonic OA. When food is placed in the osmotic agent, a water flux comes from food to the OA and a flux of solutes occurs counter-currently ${ }^{9}$. The main effect of these two

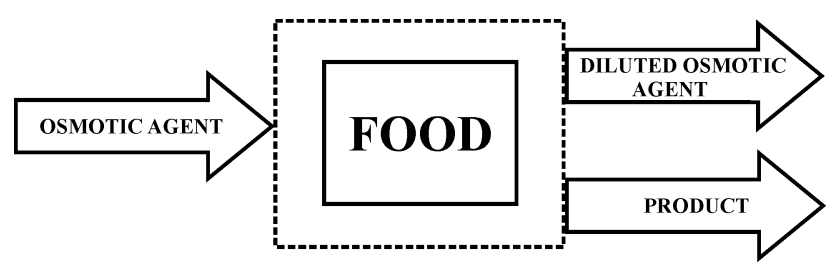

Figure 1. Scheme of osmotic dehydration process phenomena is the dilution of the OA solution, with a subsequent increase of the solution mass and the decrease of the dewatering potential. Regardless the advantages and simplicity of OD process, its wider industrial application is limited nowadays by the need of OD spent solution management. Till now the initial concentration of the osmotic agent was recovered twofold: (1) by using evaporation or (2) by adding the solute constituent. Evaporation is simple, but it is not efficient for temperature sensitive osmotic agents, like sugar solutions ${ }^{7}$. The main drawback of reconcentration by solute adding is the continuous increase of the total osmotic agent solution volume.

Currently, membrane separation techniques are fast developing processes applied also in the food processing ${ }^{10-12}$. The membrane techniques allow also for processing of very viscous solutions (e.g. concentrated sugar solutions) at moderate operation conditions (e.g. low temperature). Warczok at al. ${ }^{\mathbf{1 3}}$ investigated a direct osmosis process using nanofiltration and reverse osmosis membranes as a process of reconcentration of sugar spent solutions from osmotic dehydration of apple tissue. Warczok at al. also examined $^{\mathbf{1 4}}$ an osmotic membrane distillation process (OMD) with a microporous hydrophobic PTFE membrane for reconcentration of a pure sugar solutions. Barranco et al. ${ }^{15}$ performed OMD process for reconcentration and purification of spent brine from table olive production. All investigated membrane systems ${ }^{\mathbf{1 3}-15}$ possessed however one important drawback - the necessity of the additional post-treatment to reconcentrate the spent osmotic agent solution.

The present paper reports a study of two hybrid membrane systems based on pervaporation (PV) and/or OMD followed by PV applied for reconcentration of spent sugar solutions from osmotic dehydration and for reconcentration of diluted osmotic agent solution.

Pervaporation is a separation technique where phase change connected with mass transfer across nonporous membrane occurs. In this technique the liquid feed is in 
contact with one side of a hydrophobic membrane. Removed volatile components are evaporated on the permeate side of the membrane into a vacuum or sweeping gas. The driving force for this process is the difference in chemical potentials across the membrane ${ }^{\mathbf{1 6}}$.

The osmotic membrane distillation is a membrane contactor technique using a porous hydrophobic membrane, e.g. PTFE membrane ${ }^{\mathbf{1 7}}$, allowing the concentration of solutions at constant temperature under atmospheric pressure $^{\mathbf{1 8}}$. Both sides of the porous membrane are in contact with two aqueous solutions of different water activity, e.g. diluted OD spent solution and concentrated $\mathrm{CaCl}_{2}$ solution. Thanks to the hydrophobicity of the polymer, the membrane cannot be wetted by the liquids, thus creating vapour-liquid interfaces at each entrance of the pores. The water activity difference between the two liquid compartments translates into a vapour pressure difference at the vapour-liquid interfaces, which creates the driving force for the water transport ${ }^{17}$.

\section{EXPERIMENTAL}

\section{Pervaporation}

Pervaporation experiments were carried out in the laboratory-scale pervaporation system presented schematically in Fig. 2. The thermostated feed solution was circulating over the membrane. The permeate was collected in cold traps cooled by liquid nitrogen. During experiments the upstream pressure was maintained at the atmospheric level, while the downstream pressure was kept below $1 \mathrm{hPa}$ by using a vacuum pump ${ }^{\mathbf{1 9}}$. As the feed mixture was composed of water and a non-volatile solute (sugar or $\mathrm{CaCl}_{2}$ ), the permeate contained pure water only. The permeate flux was determined by weight, according to the following equation:

$$
\mathrm{J}=\frac{\mathrm{m}}{\mathrm{A} \cdot \mathrm{t}}
$$

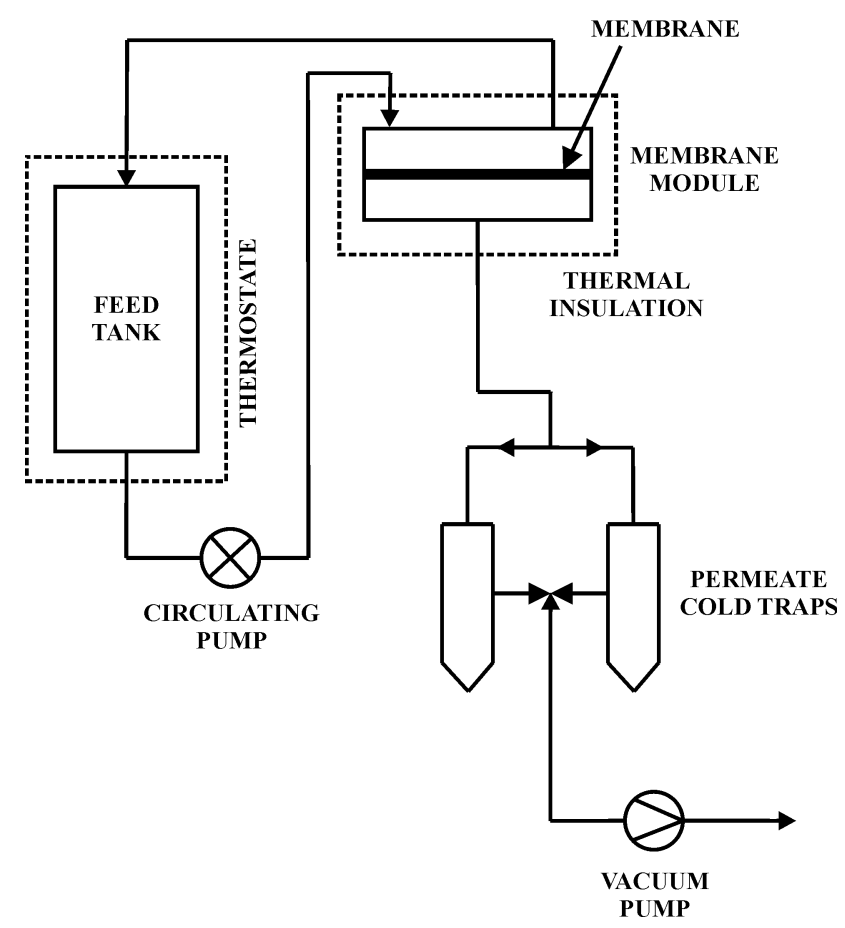

Figure 2. Scheme of pervaporation experimental set-up where $\mathrm{J}$ denotes flux $\left(\mathrm{kg} \mathrm{m}^{-2} \mathrm{~h}^{-1}\right), \mathrm{m}$ - weight of permeate $(\mathrm{kg}), \mathrm{A}-$ membrane area $\left(\mathrm{m}^{2}\right)$ and $\mathrm{t}$ - time $(\mathrm{h})$.

Three types of hydrophobic membranes were used in this study: PDMS-PAN and PDMS-PAN-NF (Pervatech, the Netherlands) and PDMS-AC. All membranes possessed a selective layer made of polydimethylsiloxane (PDMS). PDMS-PAN-NF membrane showed a slightly porous structure of the skin layer compared to PDMSPAN one. The PDMS-AC membrane was additionaly filled with active carbon particles. The effect of the non-volatile solute content on flux was determined for each membrane in contact with water-sugar solutions of concentration $0-50^{\circ}$ Brix and water-calcium chloride solutions of concentration $0-45$ wt. $\%$. Temperature during all pervaporation experiments were kept constant at $35 \pm 0.5^{\circ} \mathrm{C}$.

\section{Osmotic Membrane Distillation}

The osmotic membrane distillation set-up is presented in Fig. 3. It consists of two compartments separated by a hydrophobic, polytetrafluoroethylene porous membrane of $0.45 \mu \mathrm{m}$ pore size (PTFE 11806 membrane from Sartorius). The feed side was connected to an external tank and the stripping side was connected to a calibrated glass pipette. The stripping compartment was hermetically sealed, so the volume of solution passing through the pipette was equal to the volume of water transported through the membrane from the feed solution to the stripping one. Both solutions were constantly stirred. The OMD set-up was placed inside an oven and maintained at a constant temperature of $35 \pm 0.5^{\circ} \mathrm{C}$. The sugar solutions of concentration $30-50^{\circ}$ Brix were used as feed solutions. $50 \mathrm{wt} . \%$ calcium chloride solution was used as a stripping solution in all experiments.

AIR THERMOSTATE

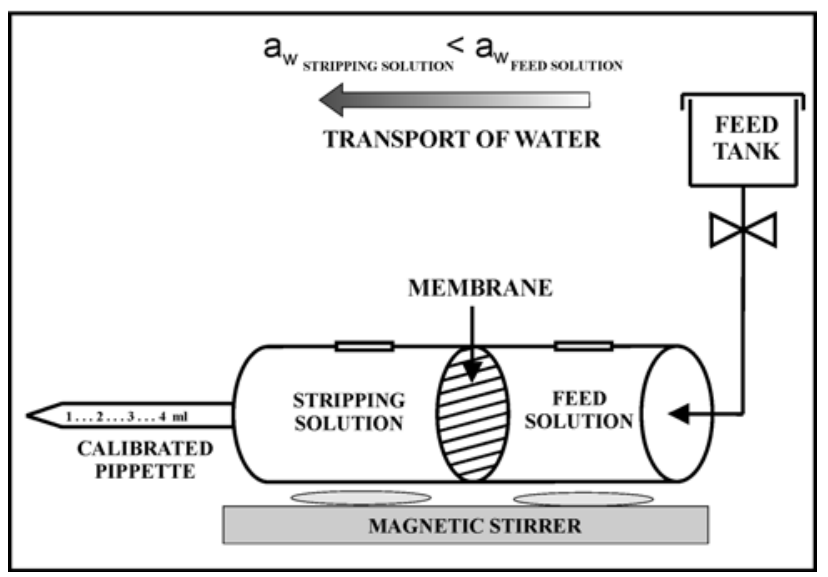

Figure 3. Scheme of osmotic membrane distillation experimental set-up

\section{Analytical measurements}

Samples collected during PV and OMD processes were analyzed using standard analytical methods ${ }^{20,21}$. Tests on the presence of sucrose in calcium chloride solution, and $\mathrm{CaCl}_{2}$ in sucrose solution from OMD processes were performed. Both tests were also used to confirm that the PV permeate is free of sucrose and calcium chloride.

Sucrose content was determined by refractometric measurements at constant temperature using Abbe refractometer RL-120. Concentration of calcium chloride solutions 
was determined using complexometric titration method with EDTA solution described in the details elsewhere ${ }^{21}$.

\section{RESULT AND DISCUSSION}

OMD-PV system for the management of sucrose and $\mathrm{CaCl}_{2}$ solutions

Fig. 4 presents the time evolution of water permeate flux during OMD process. It can be seen that for a given sugar concentration, the water permeate flux is constant up to time of 100 minutes and then steeply decreases. The decrease of water flux for time longer than 100 minutes is caused by the decreasing driving force for the OMD. It is also clearly seen that the water flux depends on the concentration of sugar solution and it increases with the dilution of sucrose solution.

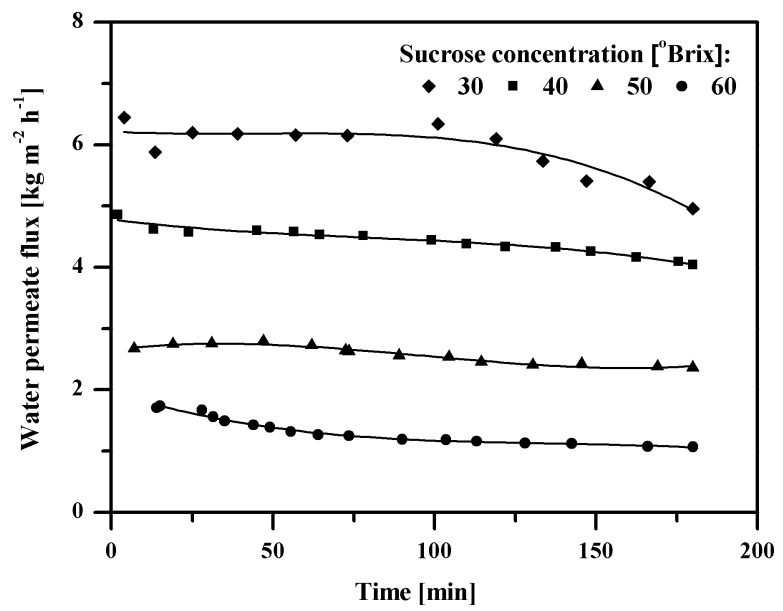

Figure 4. Effects of operation time on the OMD flux for different sucrose concentration $\left(\mathrm{T}=35^{\circ} \mathrm{C}\right)$

Obtained results (Fig. 4) allow to simulate the time profiles of the sugar reconcentration process as a function of initial sugar concentration (30, 40 and $\left.50^{\circ} \mathrm{Brix}\right)$ and the ratio $\mathrm{m}_{\mathrm{o}} / \mathrm{A}$ indicating the amount of the spent solution per membrane surface. Results of these calculations are presented in Fig. 5. It is seen that OMD process can be used for the reconcentration of sucrose solution and the efficiency of the process depends on the $\mathrm{m}_{\mathrm{o}} / \mathrm{A}$ ratio and the initial and final sucrose concentrations. Assuming the $\mathrm{m}_{\mathrm{o}} / \mathrm{A}$ ratio is equal 1:1 (Fig. 5) it was found that reconcentration of spent solution is more efficient for diluted solutions. On the other hand, for the same initial concentration, the time needed to reach the final concentration will depend on the $\mathrm{m}_{\mathrm{o}} / \mathrm{A}$ ratio. This indicates also for the flexibility of the membrane processes - with the same plant we can process different amounts of solutions, regardless initial and final concentrations.

However, the application of OMD process for reconcentration of sugar solution creates another spent stream i.e. diluted $\mathrm{CaCl}_{2}$ solution. Pervaporation was suggested to treat this stream for the reuse. First of all it was proved that the hydrophobic membrane was impermeable to $\mathrm{CaCl}_{2}$ molecules and permeate consisted of pure water only. The pervaporation flux of water in contact with $\mathrm{CaCl}_{2}$ solution is presented in Fig. 6 .

It is seen, that the permeate flux is proportional to the vapour pressure of water in feed solution. The increasing concentration of $\mathrm{CaCl}_{2}$ causes the lowering of water va-

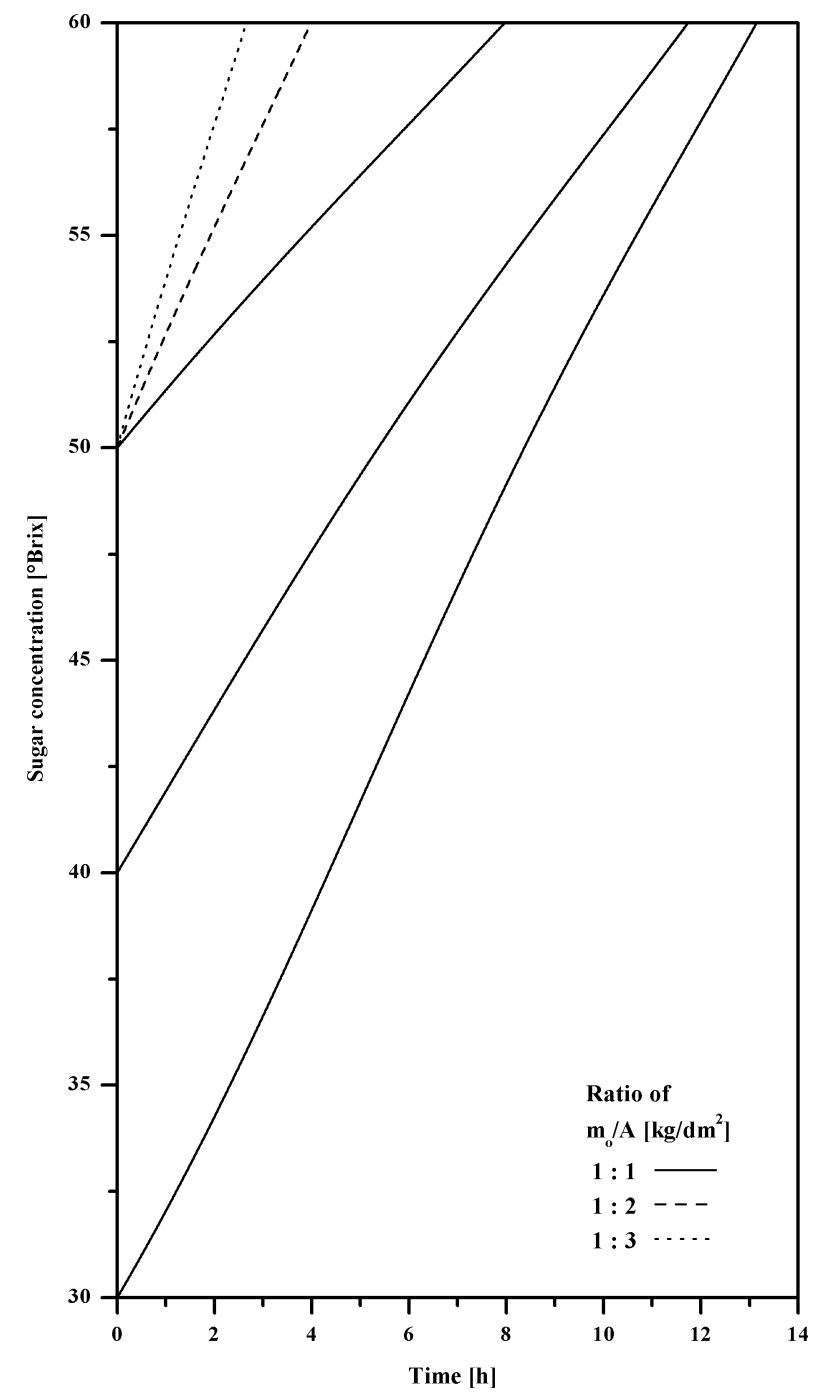

Figure 5. Calculated OMD time profiles of the sugar reconcentration process

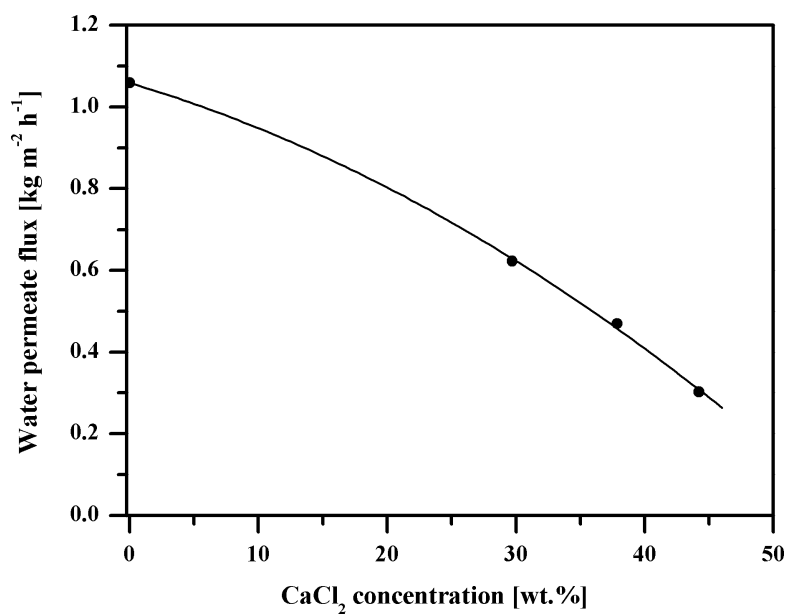

Figure 6. Relation between PV water flux and feed calcium chloride concentration for the PDMS-PAN-NF membrane $\left(\mathrm{T}=35^{\circ} \mathrm{C}\right)$

pour pressure according to the Raoult's law. This is also in the accordance with the decreasing driving force for pervaporation, which is proportional to the partial vapor pressure of volatile components in the feed ${ }^{22}$.

The results presented so far allow to suggest the following hybrid system for the management of spent sucrose solution from the osmotic dehydration of fruits (Fig. 7)

The proposed hybrid system should additionally include also a pretreatment step (e.g. membrane pretreat- 


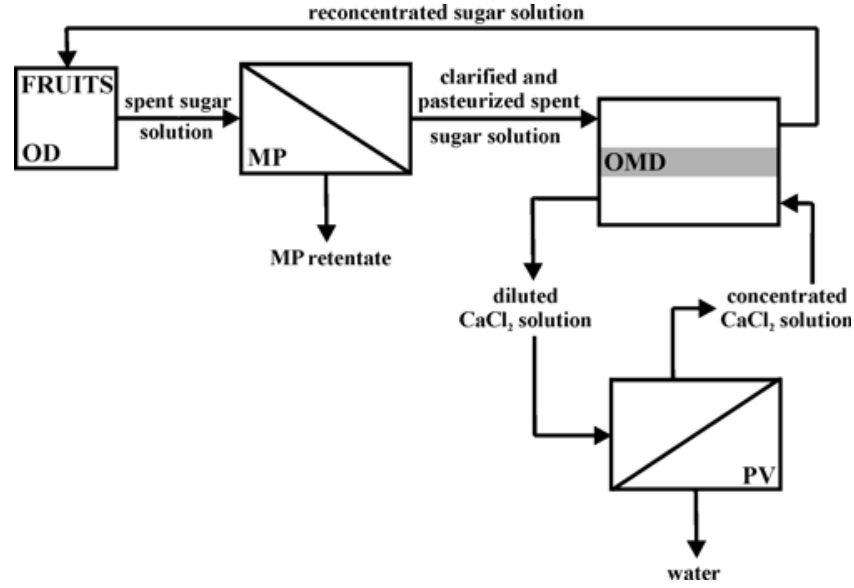

Figure 7. MP-OMD-PV hybrid system for the management of spent sugar solution from OD

ment MP) to remove the excess of solid matter created during OD. Osmotic membrane distillation would reconcentrate this pretreated diluted sugar solution which then could be directed back to the OD unit. Finally PV should process diluted $\mathrm{CaCl}_{2}$ spent solution from OMD process.

The additional calculations allowed to assess the membrane area needed in the hypothetical plant processing $1160 \mathrm{~kg}$ of spent sugar solution diluted from $60^{\circ}$ Brix to $50^{\circ}$ Brix. Calculations were made with the following assumptions: $1160 \mathrm{~kg}$ initial spent sucrose solution for OMD of $50^{\circ}$ Brix, $1000 \mathrm{~kg}$ final reconcentrated sucrose solution of $60^{\circ}$ Brix, time of the process equal to $1 \mathrm{~h}$. Based on the results presented in Figs. 6 and 7 it was possible to estimate the membrane areas needed in the OMD and PV processes. It was found that OMD modules would be equipped with $85 \mathrm{~m}^{2}$ of hydrophobic PTFE porous membranes, whereas for PV process $730 \mathrm{~m}^{2}$ of PDMS-PANNF membranes are needed.

\section{PV based system for the direct sucrose solution manage- ment}

In the previous section the hybrid system with OMDPV unit was suggested to the reconcentration of sucrose spent solution. Another possibility to solve that problem would be a system with pretreatment step directly followed by pervaporation process.

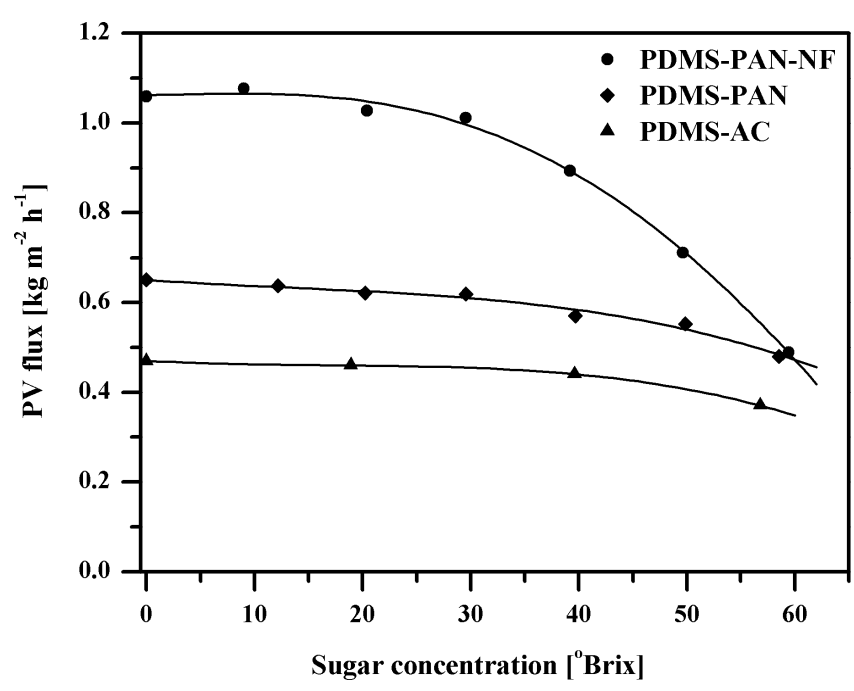

Figure 8. Relation between PV water flux and concentration of feed sugar solution in contact with PDMS-PANNF, PDMS-PAN and PDMS-AC hydrophobic membranes $\left(\mathrm{T}=35^{\circ} \mathrm{C}\right)$
The results of sucrose dehydration by pervaporation process are shown in Fig. 8. Measurements were performed for the hydrophobic dense membranes based on polidimethylsiloxane. It is seen that, similarity to pervaporation of $\mathrm{CaCl}_{2}$ aqueous solutions, PDMS-PANNF membrane produced highest permeate fluxes. This can be explained by the more open structure of the selective layer of PDMS-PAN-NF membrane. In Fig. 8 results obtained with the activated carbon filled PDMS membrane (PDMS-AC) were also presented. The PDMS-AC membrane showed the lowest transport properties what was caused by highest thickness of this membrane. It is worth noting that pervaporation of aqueous solutions of nonvolatile solutes (e.g. sugars, salts) requires the hydrophobic membrane to prevent sorption of non-volatile solutes into the membrane phase. Moreover, in the case of nonvolatile solutes, pervaporation flux is proportional to the driving force, i.e. flux should decrease with increasing concentration of feed solution (Fig. 8). It can be noticed however that the flux reduction was much higher at high sucrose concentration than it could be expected. This can be explained by the polarization effects resulting from the high viscosity of sucrose solutions.

Similarly like for the previous case, in Fig. 9 the scheme of the hybrid system for spent sugar solution from OD treatment of fruits was presented. Taking into consideration the same amount of the spent sugar solution, the size of pervaporation membranes was calculated for that hybrid system (Fig. 9). The membrane areas were $320 \mathrm{~m}^{2}$ and $380 \mathrm{~m}^{2}$ for PDMS-PAN-NF and PDMS-PAN membranes, respectively.

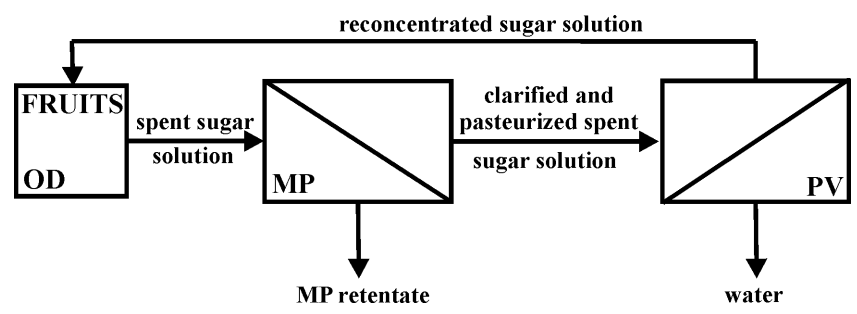

Figure 9. MP-PV hybrid system for the management of spent sugar solution from OD

\section{CONCLUSIONS}

Pervaporation of mixtures containing non-volatile solutes resulted in the removal of pure water from the system. The hydrophobic membranes applied showed good transport parameters with the PDMS-PAN-NF membrane being the most efficient one.

Osmotic membrane distillation is also an efficient method for spent sucrose solution, however during this process another side stream is generated (i.e. diluted $\mathrm{CaCl}_{2}$ ), which should be additionally treated.

Presented results proved that membrane processes like osmotic membrane distillation and pervaporation can be effectively used in the management of spent solutions. Both proposed hybrid systems could be practically applied, however direct pervaporation of sucrose solution seems to have more advantages over the other one. The membrane pretreatment step prior to the dehydration processes must be foreseen to prevent the solutions from unwanted mechanical and biological contaminations. The investigations on application of microfiltration to treat 
the OD spent solutions will be reported soon from our laboratory.

\section{ACKNOWLEDGEMENTS}

Wojciech Kujawski kindly acknowledges the research scholarship at Universitat Rovira i Virgili granted by the Catalan Government (Beques de Recerca per a Professors i Investigadors Visitants a Catalunya - 2006PIV0016).

\section{LITERATURE CITED}

1. Lewicki, P. P. (2006). Design of hot air drying for better foods. Trends Food Sci. Technol. 17, 153 - 163. DOI: 10.1016/ j.tifs.2005.10.012.

2. Di Cesare, L. F., Forni, E., Viscardi, D. \& Nani, R. C. (2003). Changes in the chemical composition of basil caused by different drying procedures. J. Agric. Food Chem. 51, 3575 - 3581. DOI: $10.1021 / \mathrm{jf} 021080$ o.

3. Marouzé, C., Giroux, F., Collignan, A. \& Rivier, M. (2001). Equipment design for osmotic treatments. J. Food Eng. 49, 207 - 221.

4. Gallart-Jornet, L., Barat, J. M., Rustad, T., Erikson, U., Escriche, I. \& Fito, P. (2007). A comparative study of brine salting of Atlantic cod (Gadus morhua) and Atlantic salmon (Salmo salar). J. Food Eng. 79, 261 - 270. DOI: 10.1016/ j.jfoodeng.2006.01.053.

5. Bellagha, S., Sahli, A., Farhat, A., Kechaou, N. \& Glenza, A. (2007). Studies on salting and drying of sardine (Sardinella aurita): Experimental kinetics and modeling. $J$. Food Eng. 78, 947 - 952. DOI: 10.1016/j.jfoodeng.2005.12.008.

6. Emam-Djomeh, Z., Djelveh, G. \& Gros, J.-B. (2001). Osmotic dehydration of foods in a multicomponent solution. Part I. Lowering of solute uptake in agar gels: diffusion considerations. Lebensm.-Wiss. u.-Technol. 34, 312 - 318. DOI: $10.1006 /$ fstl.2001.0776.

7. Dalla Rosa, M. \& Giroux, F. (2001). Osmotic treatments (OT) and problems related to the solution management. $J$. Food Eng. 49, 223 - 236.

8. Moreira, R., Chenlo, F. \& Pereira, G. (2003). Viscosities of ternary aqueous solutions with glucose and sodium chloride employed in osmotic dehydration operation. J. Food Eng. 57, 173 - 177.

9. Rastogi, N. K., Raghavarao, K. S. M. S., Niranjan, K. \& Knorr, K. (2002). Recent developments in osmotic dehydration: methods to enhance mass transfer. Trends Food Sci. Technol. 13, 48 - 59 .

10. Kumar, A., Croteau, S. \& Kutowy, O. (1999). Use of membranes for energy effcient concentration of dilute streams. Appl. Energy 64, $107-115$.

11. Cuperus, F. P. (1998). Membrane processes in agrofood. State-of-the-art and new opportunities. Sep. Purif. Technol. 14, 233 - 239.

12. Scordino, M., Di Mauro, A., Passerini, A. \& Maccarone, E. (2007). Highly purified sugar concentrate from a residue of citrus pigments recovery process. $L W T-$ Food Sci. Technol. 40, 713 - 721. DOI: 10.1016/j.lwt.2006.03.007.

13. Warczok, J., Ferrando, M., López, F., Pihlajamäki, A. \& Güell, C. (2007). Reconcentration of spent solutions from osmotic dehydration using direct osmosis in two configurations. J. Food Eng. 80, 317 - 326. DOI: 10.1016/ j.jfoodeng.2006.06.003.

14. Warczok, J., Gierszewska, M., Kujawski, W. \& Güell, C. (2007). Application of osmotic membrane distillation for reconcentration of sugar solutions from osmotic dehydration. Sep. Purif. Technol. 57, 425 - 429. DOI: 10.1016/ j.seppur.2006.04.012.
15. Romero Barranco, C., Brenes Balbuena, M., García García, P. \& Garrido Fernández, A. (2001). Management of spent brines or osmotic solutions. J. Food Eng. 49, 237 - 246.

16. Shao, P. \& Huang, R. Y. M. (2007). Review. Polymeric membrane pervaporation. J. Membr. Sci. 287, 162 - 179. DOI:10.1016/j.memsci.2006.10.043.

17. Gryta, M. (2005). Osmotic MD and other membrane distillation variants. J. Membr. Sci. 246, 145 - 156. DOI:10.1016/ j.memsci.2004.07.029.

18. Bessarabov, D. \& Twardowski, Z. (2006). New opportunities for osmotic membrane distillation. Membr. Technol. 2006, 7 - 11 .

19. Kujawski, W. \& Krajewski, S. R. (2007). Influence of inorganic salt on the effectiveness of liquid mixtures separation by pervaporation. Sep. Purif. Technol. 57, 495 - 501. DOI:10.1016/j.seppur.2006.09.020.

20. Timmermans, J. (Ed.) (1960). The Physico-chemical Constans of Binary Systems in Concentrated Solutions. Vol 4. Systems with inorganic + organic or inorganic compounds. New York, USA: Interscience Publishers Inc.

21. Meites, L. (Ed.) (1963). Handbook of Analytical Chemistry. New York, USA: McGraw-Hill Book Company Inc.

22. Chapman, P. D., Oliveira, T., Livingston, A. G. \& Li, K. (2008). Review. Membranes for the dehydration of solvents by pervaporation. J. Membr. Sci. 318, 5 - 37. DOI: 10.1016/j.memsci.2008.02.061. 